\title{
Independent Key Distribution Protocols for Broadcast Authentication
}

\author{
Bruhadeshwar Bezawada \\ Computer Science Department \\ Colorado State University \\ Fort Collins, Colorado, USA \\ bru.bezawada@colostate.edu
}

\author{
Sandeep Kulkarni \\ Computer Science Department \\ Michigan State University \\ East Lansing, Michigan, USA \\ sandeep@cse.msu.edu
}

\author{
Indrajit Ray \\ Computer Science Department \\ Colorado State University \\ Fort Collins, Colorado, USA \\ indrajit.ray@colostate.edu
}

\author{
Indrakshi Ray \\ Computer Science Department \\ Colorado State University \\ Fort Collins, Colorado, USA \\ indrakshi.ray@colostate.edu
}

\author{
Rui Li \\ Dongguan University of Technology \\ Songshan Lake, Dongguan \\ Guangdong, P.R.China \\ ruili@dgut.edu.cn
}

\begin{abstract}
Broadcast authentication is an important problem in several network settings such as wireless sensor networks and ad-hoc networks. We focus on the problem of independent key distribution protocols, which use efficient symmetric key signatures in distributed systems to permit (local) broadcast authentication. We focus on five types of communication graphs: (1) star, (2) acyclic, (3) planar, (4) complete bipartite, and (5) fully connected graphs. A star graph is the simplest network topology where a central node is transmitting authenticated broadcast messages to several satellite nodes. For star graphs, we show that as $n$, the number of satellite nodes in the star network, tends to $\infty$, it suffices to maintain $\log n+\frac{1}{2} \log \log n+1$ keys at the center node, but $\log n+\frac{1}{2} \log \log n$ keys do not suffice. We establish that this is the optimal lower bound on the number of keys for a star graph. Building on this result, we describe storage efficient key distribution for acyclic, planar, and complete bipartite graphs, when compared to existing key distribution schemes. We extend our scheme for fully connected graphs and show that it is sufficient to store $O\left(c \log ^{2} N\right)$ keys per node where $c<1$. We perform a detailed analysis of collusion resistance of our protocols and show the trade-offs against internal and external attacks depending on the size of storage. Finally, we demonstrate the practical applicability of our protocols for wireless sensor networks.
\end{abstract}

\section{KEYWORDS}

Key distribution, Broadcast authentication, Multiple Message Authentication Codes, Sensor Networks, Internet-of-Things

\section{ACM Reference Format:}

Bruhadeshwar Bezawada, Sandeep Kulkarni, Indrajit Ray, Indrakshi Ray, and Rui Li. 2018. Independent Key Distribution Protocols for Broadcast Authentication. In SACMAT '18: The 23rd ACM Symposium on Access Control Models \& Technologies (SACMAT), June 13-15, 2018, Indianapolis, IN, USA. ACM, New York, NY, USA, 12 pages. https://doi.org/10.1145/3205977.3205985

Permission to make digital or hard copies of all or part of this work for personal or classroom use is granted without fee provided that copies are not made or distributed for profit or commercial advantage and that copies bear this notice and the full citation on the first page. Copyrights for components of this work owned by others than ACM must be honored. Abstracting with credit is permitted. To copy otherwise, or republish, to post on servers or to redistribute to lists, requires prior specific permission and/or a fee. Request permissions from permissions@acm.org.

SACMAT '18, fune 13-15, 2018, Indianapolis, IN, USA

(C) 2018 Association for Computing Machinery.

ACM ISBN 978-1-4503-5666-4/18/06 . \$ \$15.00

https://doi.org/10.1145/3205977.3205985

\section{INTRODUCTION}

\subsection{Background and Motivation}

Consider a communication network represented by a graph $G=$ $(V, E)$, where $V$ is the set of nodes and $E \subseteq V \mathrm{x} V$ is a symmetric relation. We use the set of edges to denote the need for authenticity in the communication between the nodes connected by the edge. In other words, $\left(v_{i}, v_{j}\right) \in E$ implies that $v_{i}$ needs to verify authenticity of messages received from $v_{j}$ and vice versa. As an illustration, consider a sensor network consisting of a base station and a set of sensors. A sensor may need to communicate securely with a base station, where the communication may itself be assisted by other sensors in terms of routing. However, the intermediate sensors can neither learn the contents of the message nor can generate messages on behalf of the sender.

A similar scenario is a set of clients and servers, where each client needs to talk to (a subset of) the servers, but the clients (respectively, servers) do not need authentic communication among themselves. This is common in protocols defined for peer connections, such as the Border Gateway Protocol (BGP). Note that the set of edges in this graph are possibly application dependent, and may not be the same as those corresponding to physical communication links between nodes. Hence, other nodes in the network may be required for routing, although the intermediate nodes would not be able to tamper/decrypt any communication they forward.

\subsection{Problem Overview}

Now, consider the set $N B R=\left\{v_{j} \mid\left(v_{i}, v_{j}\right) \in E\right\}$. This is the set of nodes that $v_{i}$ communicates with. We consider two types of communication: unicast, where $v_{i}$ communicates with one of its neighbors and the neighbor wants to verify that the message is indeed from $v_{i}$, and (local) broadcast, where $v_{i}$ sends a message to all nodes in NBR and each node wants to verify that the message is indeed from $v_{i}$. We denote this problem as local broadcast authentication.

\subsection{Limitations of Prior Research}

Studies [39] have shown that adopting computationally intensive public-key based authentication solutions like [2, 13, 42], can significantly impact the network lifetime of networks such as sensor networks, which consist of severely resource constrained sensor nodes. Single sender broadcast authentication solutions, such as [24, 31, 32, 49], impose additional constraints like sender-receiver synchronization and packet buffering, which are not feasible in 
large distributed networks. Furthermore, these solutions do not consider different types of network topologies like complete graphs where any node can be a sender as well as a receiver.

Symmetric key based solutions [5, 11, 43], also known as Multiple Message Authentication Code (MMAC) signature schemes, have been preferred over such solutions. In these solutions, the sender maintains a pool of secret keys and each node receives a unique subset of these keys. The sender signs a broadcast message using all the pool keys and broadcasts the resulting message with the signatures to the receivers. Each receiver can independently verify only the signatures corresponding to the sub-set of keys obtained from the sender at initialization. Relatively, these solutions are more efficient than the previous solutions, but they suffer from high sender storage and broadcast communication complexity. While there have been attempts [3] to identify the lower bounds of broadcast authentication communication complexity there have been no studies on identifying the lower bounds of broadcast authentication storage complexity in MMAC schemes. Thus far, there has been no attempt to reduce the storage complexity associated with these protocols especially when dealing with large networks with different topologies like acyclic, bipartite and complete graphs.

In this work, for the first time, we address the problem of identifying lower bounds in storage complexity in symmetric key based (MMAC) broadcast authentication protocols. Our solutions achieve optimal lower bounds for star network topology and improved storage complexities for other network topologies.

\subsection{Proposed Approach}

We consider the MMAC scheme paradigm of $[5,11,43]$ and design key distribution protocols for broadcast authentication that are storage efficient. We consider five types of communication graphs: star, acyclic, planar, complete bipartite, and fully connected graphs. Of these, the star graph is motivated by examples such as the sensor networks scenario discussed. A planar graph is motivated by the case where nodes are distributed in a plane and a planar communication network is established for connectivity. Acyclic and complete bipartite graphs are useful in cases of client-server applications. Finally, fully connected graphs capture the case where each node may be interested in authentic communication from all other nodes in the network.

Based on these network models our approach is as follows. First, we focus on designing key distribution protocols for the star graph: a center node acting as a sender connected to several satellite nodes, which are the receivers. Our key distribution protocol ensures that the sender has the smallest possible pool of keys, and that the sender can distribute a unique subset of keys, chosen from this pool, to each receiver. Next, we use the low storage achieved in the star graph to design key distribution protocols for planar, acyclic, complete bipartite, and fully connected graphs. By leveraging the topology features of these varied graphs, we reduce the storage cost, as well as, the signature generation and verification costs for the nodes in these graphs.

\subsection{Technical Challenges and Solutions}

The first challenge is that, the keys assigned to nodes need to be independent, i.e., one node cannot derive a key stored by another node. Independent keys permit asynchronous and periodic update where only one key is changed as needed. For example, a simple approach for such asynchronous update is to encrypt the new key with the corresponding old key and transmit it. But if some other node had to maintain a key that is dependent on $k$, say a hashed version of $k$, then the new key needs to be changed any time $k$ is changed. In our solution, we ensure that the keys are independent by choosing the sender pool of keys uniformly at random and not imposing any relationship between any pair of keys. Each receiver gets a unique subset from this pool of keys, and therefore, the node keys are independent as well.

The second challenge is that, the cost of the broadcast communication should be proportional to the keys stored by the sender and the receivers in the communication graph. For example, in complete graphs, where every node is a sender as well as a receiver, it is quite essential to ensure that each receiver maintains only a small number of verification keys from other senders besides the keys it has to maintain as a sender. To address this, we focus on the star communication graphs and identify an optimal lower bound for the storage required at the sender and the receivers. We show that as $n$, the number of satellite nodes in the star network, tends to $\infty$, it suffices to maintain $\log n+\frac{1}{2} \log \log n+1$ keys at the center node, which is a tight bound. Furthermore, as the keys are independent, our result significantly reduces the signature generation cost for the sender and the verification cost for the receivers.

The third challenge is that the network may be organized in different topologies such as acyclic, planar, complete bipartite and fully connected graphs. The key distribution protocol should ensure that the node storage depends asymptotically on the size of the network for every kind of topology. To solve this, we compose the complex network topologies as instances of star graphs and achieve the desired key distribution suitable for broadcast authentication.

Finally, since the key distribution protocols in the MMAC paradigm require that the keys are shared across multiple receivers, it is essential to address the issue of collusion among receivers. We address this by varying the different parameters of our key distribution protocol and demonstrate the collusion resistance achieved by each instance of the protocol.

\subsection{Key Contributions}

Our key contributions are: (1) We show that for a star graph with $n$ satellite nodes, there exists a protocol that maintains $\log n+$ $\frac{1}{2} \log \log n+1$ keys at the center node, which is the optimal storage. This condition holds in virtually all network sizes of practical interest. Also, under the assumption that the number of keys maintained by the satellite nodes is identical, we show that there does not exist a protocol that maintains $\log n+\frac{1}{2} \log \log n$ keys at the center node. (2) We show that our protocols can also be extended to acyclic, planar, complete bipartite, and fully connected graphs. (3) We show that the protocol can provide higher collusion resistance than the existing protocols under different attack scenarios. (4) We show that our protocols provide tradeoff between internal and external attackers. (5) We demonstrate the applicability of our protocols in wireless sensor networks with promise for Internet-of-Things.

\section{PROBLEM STATEMENT}

First, we state the problem of authentication under any communication model, and then, state the problem of authentication ${ }^{1}$ with broadcast capability and without broadcast capability.

${ }^{1}$ Unless explicitly stated, we use the terms "authentication", "authenticity" and "source authentication" interchangeably. 
The Authentication Problem. For a message $m$ transmitted from a source $A$ to a destination $B$;

Message Authenticity: $B$ should be able to verify that the source of the message is indeed $A$ and,

Message Integrity : $B$ should be able to verify that the message integrity has not been compromised in transit.

Threat Model. We consider impersonation as the main threat, i.e., one node being able to authenticate as another node. We focus on key distribution in which a single node, using the set of stored keys, cannot impersonate another node in the network.

Problem Statement Motivation. To motivate the problem, we begin with star networks where there is one center node and other satellite nodes that only communicate with the center node. Our problem statement first focuses on defining the number of stored keys and then relates it to the cost of broadcast authentication. To motivate the need for such a definition, consider the following solution for key distribution in a star network.

The center node maintains a key $x$. and each satellite node has an ID ranging from $1, \cdots, n$. The key associated with satellite node with ID $j$ is $f(x, j)$ where $f$ is a one-way function. Clearly, $f(x, j)$ could be used for unicast communication to/from $j$ and the center node. For example, $f(x, j)$ could be used to generate a message digest that the receiver can verify. Since $f(x, j)$ is known only to the center node and satellite node $j$, only they can generate the corresponding message digests. In this solution, the number of keys that each node stores is 1 . While the center node has access to many keys that it can generate at run-time, they need not be stored. Thus, we need to distinguish between the number of stored keys and the number of generated keys.

A node can store two sets of keys: those that it distributes to other nodes and those that it receives from other nodes. We denote the keys stored by node $v_{i}$ as $\operatorname{stored}\left(v_{i}\right)$. A node can generate keys from the $\operatorname{stored}\left(v_{i}\right)$ using any operation like, $X O R$, one-way hashing and so on, which can result in a potentially unbounded set of keys. We denote the set of all such operations by, gen and the set of all such keys that can be generated as, generated $\left(v_{i}\right)$. Observe that $\operatorname{stored}\left(v_{i}\right) \subseteq$ generated $\left(v_{i}\right)$. Based on this, we make a standard assumption about the adversary capability, i.e., the adversary can combine the keys it has, e.g., by XORing them/adding them, oneway hashing and so on, to generate newer keys. However, such combination does not allow the adversary to guess the keys that other nodes have.

While the above solution works for unicast, it is very inefficient for broadcast. For broadcast, the center node must provide a separate message authentication code using $f(x, j) \forall j$ where $1 \leq j \leq n$. Each satellite node can verify the authentication code by generating the code locally, using its key $f(x, j)$, and comparing the generated code with the code sent by the center node. Clearly, in this solution, the cost of generating authentication codes, which is $O(n)$, for broadcast is very high.

Now, consider the following scenario. Given two nodes, $j$ and $k$ where $j$ has key $s$ and $k$ has key $f(s)$ where $f$ is some function, a message encrypted with $s$ cannot be decrypted by $k$ (unless strong requirements are added for function $f$ and the algorithm for encryption). Since we would like to allow the use of any symmetric key based encryption approaches, for $j$ and $k$ to communicate securely, they must share common key(s). As a result, for broadcast communication, where the sender signs the message separately with multiple keys, the receiver must have one or more of the keys that are actually used for generating the signature. Therefore, to provide broadcast authentication capability, we impose the following constraint on the problem of key distribution in star network: We require that a sender maintain a set of keys. Whenever the sender sends a (local) broadcast message to its neighbors, it provides authentication codes using (a subset of) of the keys it has. Upon receiving the message, the receiver will verify the signatures it can verify. Note that the receiver cannot verify all signatures since it does not have all the keys used by the sender. It is required that when the receiver verifies the signatures it can, it must be the case that the message is indeed authentic.

Based on this discussion, we now clearly define the problem statements for message authentication that distinguish between unicast and broadcast capability with differing key storage scenarios. These statements allow us to describe the primary problem statement of this work, i.e., independent key distribution for broadcast authentication.

Problem Statement 2.1 (Key Distribution With Broadcast Capability). Given an undirected graph $G(V, E)$ where $V$ is the set of nodes and $E$ is the set of edges such that given two nodes $v_{i}$ and $v_{j}$ in $V,\left(v_{i}, v_{j}\right) \in E$ iff $v_{i}$ needs the communication with $v_{j}$ to be authentic. Then, assign to each node $v_{i}$ a set of keys, $\operatorname{stored}\left(v_{i}\right)$, such that for any $\left(v_{i}, v_{j}\right) \in E$ :

1) (Existence of Common Keys usable for broadcast): $\operatorname{stored}\left(v_{i}\right) \cap$ generated $\left(v_{j}\right) \neq \phi$. where generated $\left(v_{j}\right)$ is the set of keys that can be generated from $\operatorname{stored}\left(v_{j}\right)$ (subject to constraints specified later).

2) (Authenticity): For any $v_{k} \in V: v_{k} \neq v_{i} \wedge v_{k} \neq v_{j}$ : $\left(\operatorname{stored}\left(v_{i}\right) \cap\right.$ generated $\left.\left(v_{j}\right)\right) \nsubseteq$ generated $\left(v_{k}\right)$.

Note that this problem statement requires broadcast capability, i.e., if a node, say $v_{i}$, sends a message and includes a signature block that contains a signature from each of the key it stores then any node that receives this message can verify the signatures by using the keys in $\operatorname{stored}\left(v_{i}\right) \cap$ generated $\left(v_{j}\right)$. If all signature verifications succeed then the message is indeed from $v_{i}$. Thus, the cost of broadcast authentication is proportional to $\operatorname{stored}\left(v_{i}\right)$. This implies that reducing the cost of broadcast authentication is equivalent to reducing the size of $\operatorname{stored}\left(v_{i}\right)$.

Problem Statement 2.2: (Key Distribution Without Broadcast Capability.) Given an undirected graph $G(V, E)$ where $V$ is the set of nodes and $E$ is the set of edges such that given two nodes $v_{i}$ and $v_{j}$ in $V,\left(v_{i}, v_{j}\right) \in E$ iff $v_{i}$ needs the communication with $v_{j}$ to be authentic. Then, assign to each node $v_{i}$ a set of keys, $\operatorname{stored}\left(v_{i}\right)$, such that for any $\left(v_{i}, v_{j}\right) \in E$ :

1) (Existence of Common Keys): $\operatorname{generated}\left(v_{i}\right) \cap \operatorname{generated}\left(v_{j}\right) \neq$ $\phi$.

2) (Authenticity): For any $v_{k} \in V: v_{k} \neq v_{i} \wedge v_{k} \neq v_{j}$ : $\left(\right.$ generated $\left(v_{i}\right) \cap$ generated $\left.\left(v_{j}\right)\right) \nsubseteq$ generated $\left(v_{k}\right)$.

Lemma 2.2.1. Given any protocol that satisfies Problem Statement 2.1, there is an equivalent protocol that satisfies Problem Statement 2.2.

Proof. Let $p$ be the protocol that satisfies 2.1. Let $\operatorname{stored}_{p}(v)$ denote the secrets stored at $v$ in protocol $p$. Let $\operatorname{generated}_{p}(v)$ denote the secrets that can be generated at node $v$ by using the secrets $\operatorname{stored}_{p}(v)$. Let $p^{\prime}$ be a protocol such that $\operatorname{stored}_{p^{\prime}}(v)=$ $\operatorname{generated}_{p}(v)$, where stored $_{p^{\prime}}(v)$ denotes the secrets stored at $v$ in protocol $p^{\prime}$. It is straightforward to observe that $p^{\prime}$ satisfies the constraints of Problem 2.2

Any shared key protocol that provides authentication meets the constraints of Problem Statement 2.2. Protocols that do not satisfy it 
include those that use asymmetric keys [2,13, 42], physical security [36] and delayed authentication approaches [24, 31, 32, 49]. From Lemma 2.2.1, it follows that for any protocol that satisfies key distribution without broadcast capability, there is a corresponding protocol that provides broadcast capability. However, the protocol generated by application of this Lemma is likely to maintain a substantially larger number of keys than the original protocol. Since our goal is to reduce the keys stored at a given node, it follows that it is desirable to develop a protocol that satisfies Problem Statement 2.1. Next, we state the problem of independent key distribution for unicast.

Problem Statement 2.3: (Independent Key Distribution for Unicast) Given an undirected graph $G(V, E)$ where $V$ is the set of nodes and $E$ is the set of edges such that given two nodes $v_{i}$ and $v_{j}$ in $V,\left(v_{i}, v_{j}\right) \in E$ iff $v_{i}$ needs the communication with $v_{j}$ to be authentic. Then, assign to each node $v_{i}$ a set of keys, $\operatorname{stored}\left(v_{i}\right)$, such that for any $\left(v_{i}, v_{j}\right) \in E$ where $v_{i} \neq v_{j}$ :

Independent Common Keys: $\operatorname{stored}\left(v_{i}\right) \cap \operatorname{generated}\left(\operatorname{stored}\left(v_{j}\right)-\right.$ $\left.\operatorname{stored}\left(v_{i}\right)\right)=\phi$.

Authenticity: For any $v_{k} \in V: v_{k} \neq v_{i} \wedge v_{k} \neq v_{j}:\left(\operatorname{generated}\left(v_{i}\right) \cap\right.$ $\left.\operatorname{generated}\left(v_{j}\right)\right) \nsubseteq \operatorname{generated}\left(v_{k}\right)$.

The notion of independent common keys captures the intuition that the keys stored or generated at $v_{i}$ and $v_{j}$ are truly independent of each other. The above problem statement allows us to explore solutions where refreshing or updating the keys is possible. For example, we can update a particular key in the key distribution and send it to those nodes who need that key. Removing an independent key will not affect the key sharing patterns among the nodes and therefore, we can be assured of retaining the authentication properties of the key distribution protocol that contains only independent keys. This is not possible in key distribution protocols such as [31] that rely on generated keys to achieve authentication.

Theorem 2.3.1 Let $p$ be a key distribution that satisfies problem statement 2.1 (respectively, 2.2) and 2.3. Let $k$ be one of the keys used in the distribution. Let $k^{\prime}$ be a new key which is not currently used in $p$. Now, if we replace all instances of $k$ with $k^{\prime}$, then the corresponding key distribution also satisfies problem statement 2.1 (respectively, 2.2) and 2.3.

Proof. Note that the common keys in $p$ are independent of each other. This implies that changing these keys will not affect the common keys among any other nodes in the system. Hence, replacing a key in $p$ will result in a key distribution where the corresponding nodes share a new common key and no other keys among the other nodes are affected by this change. This proves that the changed key distribution satisfies the problem statement 2.3 .

Theorem 2.3.1 shows that if the keys are independent then it is possible to change one key without affecting other keys. If the keys are not independent then this is not guaranteed. For example, if node $v_{i}$ maintains a key $k$ and node $v_{j}$ maintains $f\left(k, I D_{v_{j}}\right)$ where $f$ is a one-way hash function then just changing $k$ to $k^{\prime}$ is not possible as it requires the key maintained at $v_{j}$ to be updated simultaneously. This problem becomes even more challenging if such hashed keys are maintained at several nodes. The independence feature is especially valuable if the key distribution utilizes a large number of keys and changing several keys in a coordinated fashion is difficult. Thus, independence allows keys to be effectively refreshed. For example, to refresh key $k$ with $k^{\prime}$, one can just send $k^{\prime}$ by encrypting it with $k$. Finally, we state the problem of independent key distribution with broadcast capability, which is the focus of our work.
Problem Statement 2.4: Independent Key distribution with broadcast capability Given an undirected graph $G(V, E)$ where $V$ is the set of nodes and $E$ is the set of edges such that given two nodes $v_{i}$ and $v_{j}$ in $V,\left(v_{i}, v_{j}\right) \in E$ iff $v_{i}$ needs the communication with $v_{j}$ to be authentic. Then, assign to each node $v_{i}$ a set of keys, denoted by $\operatorname{stored}\left(v_{i}\right)$, such that for any $\left(v_{i}, v_{j}\right) \in E$ where $v_{i} \neq v_{j}$, all the constraints of Problem Statements 2.1 and 2.3 are satisfied. We focus on this problem in our work and describe our solutions.

One of the main reasons for describing these problems is to clearly distinguish among the storage costs required for solving the authenticity problem across different communication capabilities and to establish the notion of optimal storage for key distribution. For instance, a shared key distribution protocol that solves the authenticity problem in 2.1 can solve Problem Statement 2.2, but might require a higher number of keys, because additional keys need to be generated. For instance, stored $\left(v_{i}\right) \cap \operatorname{generated}\left(v_{j}\right) \neq \emptyset$ implies that generated $\left(v_{i}\right) \cap \operatorname{generated}\left(v_{j}\right) \neq \emptyset$. But as shown in proof of Lemma 2.2.1, the storage required by the latter expression is higher. One other reason for these definitions is to clearly demarcate the storage capabilities of solutions proposed in literature so far.

\section{RELATED WORK}

Secure communication using symmetric key mechanisms has been widely studied. For fully connected network topologies, it has been shown by $[1,4,6,7,12,14,16,21,23,28,29,44,45,47]$, that the number of keys stored by each node can be less than $O(n)$ for a system of $n$ nodes. In $[14,21]$, the authors describe a key distribution protocol which allow any two nodes to communicate securely while storing only $O(\sqrt{n})$ keys. These protocols are designed for the case where the communication graph is fully connected. Further, in [1], the authors describe a key distribution protocol that requires each node to store only $O\left(\log ^{2} n\right)$ keys. They show the existence of $O(\log n)$ key distribution protocol for star network like scenario without actually constructing the protocol.

Reducing the storage to logarithmic bounds has benefits in resource constrained networks such as sensor networks, ad-hoc networks and IoT networks. Examples of such resource constrained networks include sensor networks [12,31], ad-hoc networks [18, 25] and IoT [17, 20, 35, 38, 51]. In [17], the authors discuss about the need for application data authenticity and confidentiality in IoT networks. However, in these works $[1,4,6,14,21,23,27,28,34$, $45,46,48]$, pair-wise confidentiality, not broadcast authentication, was the main focus. Nevertheless, these protocols can be extended for authentication by, possibly, using additional storage so that they satisfy the constraints of the authentication problem. For comparison with our approach, we have considered such extensions for the low storage schemes in $[1,11,28]$ and demonstrate that our approach achieves the lowest possible storage compared to these schemes (cf. Section 5.1). Also, we have not considered the problem of confidentiality in broadcast communication [41] as it is orthogonal to the problem in this paper. While the focus of our work is on deterministic key distribution schemes, such as those mentioned above, there is significant work in probabilistic random key distribution schemes such as [7, 12, 47].

Solutions like [10] are not suited to solve Problem statement 2.1 as they do not have broadcast capability. In [5], the authors describe a probabilistic symmetric key distribution protocol, based on the results from [10], that satisfies the Problem statement 2.1. 
For fully connected networks, the solution in [5] proposes a key distribution which separates senders and receivers. However, this solution does not satisfy the constraints of Problem statement 2.4. The keys distributed to the receivers are a function of the keys given to the senders to enable appropriate sender authentication. Alternatively, they describe an approach where the number of keys held by each node depends on the desired collusion resistance $(w)$ and the acceptable probability of forging the signatures $(q)$ and is quantified by: $O(e(w+1) \ln (1 / q)$. However, to achieve a low value of $q$, the number of sender signatures and consequently, the number of sender keys needs to be increased considerably. Another drawback in [5] is that the time taken to verify whether the key distribution has the desired property is $O\left(n^{3}\right)$ for a group of $n$ nodes, which is computationally intensive for large groups, say, $n>20000$ and if the keys need to refreshed periodically. In contrast, our protocols are deterministic and the key distributions can be generated using standard combinatorial enumeration techniques [9].

In [11], for authentication with broadcast capability, the authors describe a logarithmic key distribution protocol for a star network where the storage is logarithmic for both the center, which stores $2 \log n$ keys and the satellite nodes, which store $\log n$ keys. They extend their results to achieve logarithmic key distribution for several classes of communication networks including, star networks, acyclic networks, cycle-limited networks, planar networks and dense bipartite networks. We show that the storage used in their approach is higher than what is required in our approach and our scheme supports a larger receiver base for the same sender storage while achieving higher collusion resistance for similar storage. A major difference between [11] and our work is that, we have considered fully connected networks and describe efficient key distribution protocols, which require each node to store a small number of keys, i.e., poly-logarithmic in the size of the group.

In [31], and later in extended works like [24, 49], the authors describe broadcast authentication schemes based on one-way hash chains. The sender reveals values from a one-way hash chain at appropriate time intervals periodically. Each node verifies the authenticity of these values using a master hash value which serves as a public-key. Their scheme works essentially for star networks and requires an initialization step for every new session, i.e., setting up of a new master hash value. Such protocols, using generated keys, might appear to have the best storage solution, i.e., requiring only one (generated) key per receiver, but updating this key across sessions takes considerable effort for the sender and requires additional effort in synchronizing sender and receiver. Our solutions apply for the paradigm of Multiple Message Authentication Codes (MMAC) based solutions using independent shared keys and we focus on the optimal solution in this approach. We emphasize the notion of independent keys due to the additional security properties they provide such as updating the keys efficiently.

In [3], Boneh et al., discuss in depth regarding the lower bounds of broadcast authentication communication complexity. However, this work did not explore the storage complexity and the correlation between these two complexities. In [15], the authors explore the constructions of [5] to authenticate messages in the controller area network (CAN) broadcast serial bus for in-network communication in modern day vehicles. In such scenarios, our protocols will provide considerable storage savings and communication efficiency due to the real-time constraints in these networks. Recent efforts in diverse networks like vehicular ad-hoc networks (VANETS), e.g., in [8] have explored this problem with the help of an online centralized key distribution center, which is may not always be possible.

Several solutions $[2,19,22,26,33,40,41,50]$ exist for achieving broadcast confidentiality, i.e., these protocols concern with the establishment of a single group wide secret to secure all communications in the group. These protocols can handle dynamic group membership wherein nodes can join and leave the group, i.e., these protocols establish a new group secret in an efficient manner. There are two important differences between the solutions we describe and these solutions. First, in these solutions, confidentiality of communication is the main concern and the group wide secret is available with any group member, which makes it difficult to authenticate the sender of a particular message and might need public-key mechanisms like RSA signatures or [31, 42] to achieve source authentication. In our work, we focus on the ability of receiver to authenticate the sender of any message without ambiguity. Second, there is usually the presence of single group wide controller who handles the dynamic membership changes and re-establishes new secrets among the group members. In our work, the group controller is not assumed to be available at all times. Node additions and revocations are done by the controller in an off-line manner. More importantly, the design of our protocols ensures that nodes can be added to the group without having to change the keys across the entire group.

\section{OPTIMAL SOLUTION FOR STAR NETWORK}

In this section, we focus on key distribution for a star network, i.e., a center node and a set of satellite nodes that communicate with it. Let the set of keys at the center node be $K$ and $|K|=k$. Each satellite node receives a unique subset of size $l, l>0$, from this set. Note that, by construction, given any two distinct satellite nodes $v_{i}, v_{j}$, we have $K \cap \operatorname{generated}\left(v_{i}\right) \nsubseteq$ generated $\left(v_{j}\right)$. Thus, the constraints of Problem 2.1 are satisfied. We term this protocol instance as $p(k, l)$. Technically, $p(k, l)$ is a family of protocols. However, for brevity of presentation, we denote $p(k, l)$ as a protocol. We note that all the results in this paper attributed to protocol $p(k, l)$ are valid for any member of the $p(k, l)$ protocol family. Using $p(k, l)$, authentication can be achieved in the communication as follows.

(1) To authenticate a message $m$ broadcast by the center node to the satellite node, the center node generates authentication codes with each of the $k$ keys. Each authentication code consists of the message digest $m d$ of the message computed using a key held by the center. The center appends the $k$ authentication codes thus generated to the message and broadcasts the resulting message. Now, when a satellite node receives this message, it uses its subset of $l$ keys to compute $l$ authentication codes. The satellite node then verifies these authentication codes with the corresponding authentication codes sent by the center node. Note that, each satellite node can verify only those authentication codes for which it has the corresponding generating key.

(2) To authenticate a unicast message $m$ from the center to a particular satellite node, the center node first computes an $X O R$ of the subset of the keys that this satellite node knows. Now, the center node uses the combined key to compute the message authentication code for this message. The center node appends this authentication code to the message and unicasts the message to the satellite node. The satellite node $X O R s$ its subset of keys and uses the generated key to verify the authentication code sent by the center node. Due to the 
independence of keys, using XOR to combine the secrets to the secret to authenticate a message provides informationtheoretic security under assumption of no collusion.

(3) To authenticate a unicast message $m$ from a satellite node to the center, the satellite node uses the same approach used by the center node for authenticating unicast messages.

An example distribution is shown in Figure 1, where a center $A$ distributes keys to the satellite nodes $A_{1}, A_{2}, \ldots, A_{6}$. By design, the set of keys given to each node is unique and hence, can be used to authenticate messages sent to that node.

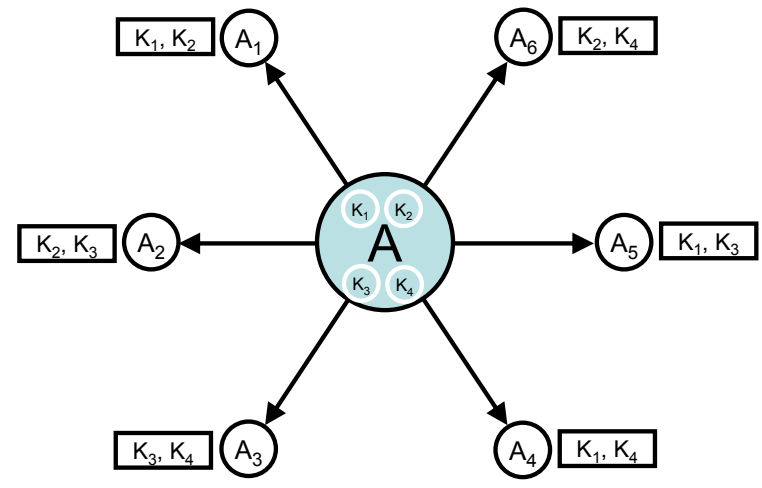

Figure 1: Example key distribution for star network

In the remaining section, we consider the case where the number of satellite nodes, $n$, tends to $\infty$ : First, we show (cf. Theorem 4) that $\log n+c \log \log n$ keys do not suffice if $c<1 / 2$. Using the proof of this result, we show (cf. Theorem 5) that $\log n+\frac{1}{2} \log \log n+1$ keys suffice to handle $n$ nodes whereas $\log n+\frac{1}{2} \log \log n$ do not.

Theorem 1. $p(k, l)$ solves Problem 2.4 in the star network.

Proof. The proof follows from the construction of $p(k, l)$. $\quad \square$

Theorem 2. Protocol $p(k, l)$ can accommodate up to $C(k, l)$ satellite nodes where $C(k, l)$ is the number of unique subsets of size $l$ chosen from the pool of $k$ keys.

Proof. This follows directly, as this $C(k, l)$ is the number of unique subsets of keys that can be generated.

Corollary 3. For a given value of $k$, choosing $l=k / 2$ maximizes the number of satellite nodes that can be accommodated.

Lemma 4. As $n \rightarrow \infty$, protocol $p(k, l)$, where $k=\log n+$ $\log \log n$ and $l=k / 2$, provides authentication for the star network with $n$ nodes.

Proof. To prove this, we show that, when $k=\log n+\log \log n$ and $l=k / 2,{ }^{2}$ the number of unique subsets of keys that are possible, i.e., $C(k, l) \geq n$. This implies that each satellite node receives a different subset of keys and hence, based on Theorems 1 and 2, $p(k, l)$ can be used for authentication in star network.

We use Stirling's approximation (originally attributed to Abraham de Moivre) [30, 37] for factorials which states that, for large values of $n, n ! \approx(n / e)^{n} \cdot \sqrt{2 \pi n}$. Now, $C(k, l)=\frac{k !}{(l) !(k-l) !}$. Since $l=k / 2$, this values simplifies to: $\frac{k !}{(k / 2) !(k / 2) !}$. Now, using Stirling's approximation, we have

$$
C(k, k / 2)=\frac{k !}{(k / 2) !(k / 2) !}
$$

${ }^{2}$ Since we are dealing with large values of $n$, for simplicity of presentation, we omit the floor/ceiling operations required in computing $\log n, l$, etc.

$$
\begin{aligned}
& \approx \frac{(k / e)^{k} * \sqrt{2 \pi k}}{\left((k / 2 e)^{k / 2} \sqrt{2 \pi(k / 2)}\right)^{2}} \\
& =\frac{2^{k} \cdot 2}{\sqrt{2 \pi k}}
\end{aligned}
$$

Now, letting $k=(\log n+\log \log n)\}$

$$
\begin{aligned}
& =\frac{2^{(\log n+\log \log n)} 2}{\sqrt{2 \pi(\log n+\log \log n)}} \\
& =n \frac{2 \log n}{\sqrt{2 \pi(\log n+\log \log n)}}
\end{aligned}
$$

and after canceling out common terms we obtain the following term:

$$
\frac{2.2^{(\log n+\log \log n)}}{\sqrt{(2 \pi \log n+\log \log n)}} \geq \frac{2 n \log n}{\sqrt{(2 \pi 2 \log n)}}=n \cdot \sqrt{\log n} / \sqrt{4 \pi}
$$

Now, as $n \rightarrow \infty$, the multiple of $n$ tends to $\infty$. Hence, as $n \rightarrow \infty$, $C(k, k / 2)>n$, where $k=\log n+\log \log n$. In other words, the number of subsets generated by choosing $k=\log n+\log \log n$ and $l=k / 2$ is greater than or equal to $n$. Thus, this key distribution ensures authentication in the star network.

Theorem 4. As $n \rightarrow \infty$, protocol $p(k, l)$ where $k=(\log n+$ $c \log \log n)$, where $c<1 / 2$ and $l=k / 2$ cannot provide authentication for the star network with $n$ nodes.

Proof. We evaluate $C(k, l)$ using Stirling's approximation and show that this value is less than $n$. This implies that there will at least two nodes who might receive the same subset of keys. Based on Theorems 1 and 2, this result proves this theorem. From the above proof,

$$
\begin{aligned}
C(k, k / 2) & =\frac{k !}{(k / 2) !(k / 2) !} \\
& \approx \frac{(k / e)^{k} * \sqrt{2 \pi k}}{\left((k / 2 e)^{k / 2} * \sqrt{2 \pi(k / 2)}\right)^{2}} \\
& =\frac{2^{k} * 2}{\sqrt{2 \pi k}}
\end{aligned}
$$

\{Using this approximation\}

$$
C(k, k / 2) \approx \frac{2^{k} * 2}{\sqrt{2 \pi k}}
$$

\{Now, letting $k=\log n+c \log \log n$ \}

$$
\begin{aligned}
& =\frac{2^{(\log n+c \log \log n)} * 2}{\sqrt{2 \pi(\log n+c \log \log n)}} \\
& =n \frac{2.2^{c \log \log n}}{\sqrt{2 \pi(\log n+c \log \log n)}} \\
& =n \frac{2 \cdot\left(2^{\log \log n)^{c}}\right.}{\sqrt{2 \pi(\log n+c \log \log n)}} \\
& =n \frac{2 \cdot(\log n)^{c}}{\sqrt{2 \pi(\log n+c \log \log n)}}
\end{aligned}
$$

This implies that there will be at least two nodes who might receive the same subset of keys. Substituting for the values of $k, l$ in $C(k, l)$, where $k=(\log n+c \log \log n), c<1 / 2$ and $l=k / 2$, and using Stirling's approximation, we obtain:

$$
\frac{2.2^{(\log n+c \log \log n)}}{\sqrt{(2 \pi \log n+c \log \log n)}}
$$

This term simplifies to:

$$
\frac{2 n \cdot \log n^{c}}{\sqrt{(2(\pi \log n+c \log \log n))}} \leq \frac{2 n(\log n)^{c}}{\sqrt{(4 \pi \log n)}}=\frac{n}{(\pi \log n)^{1 / 2-c}}
$$

Note that, as $n \rightarrow \infty$, the multiple of $n$ in the above formula tends to 0 , where $c<1 / 2$, this value is always smaller than $n$ 
due to the presence of a non-negligible denominator. Thus, for $k=(\log n+c \log \log n), c<1 / 2$ and $l=k / 2$, the value $C(k, l)$ generates less than $n$ unique subsets of keys. Hence, this $p(k, l)$ does not ensure authentication in star network. Therefore, as $n \rightarrow \infty$, $C(k, k / 2)<n$.

Based on the above theorem, if $c=1 / 2$ then the multiple of $n$ is $\frac{2}{\sqrt{2 \pi}}$. Since this number is less than $1, \log n+c \log \log n$ keys do not suffice when $c=1 / 2$. However, if the number of keys is $\left(\log n+\frac{1}{2} \log \log n+1\right)$, then in the above formula, the multiple of $n$ is $\frac{4}{\sqrt{2 \pi}}$. Since this number is greater than $1,\left(\log n+\frac{1}{2} \log \log n+1\right)$ keys suffice for key distribution in a star network as $n \rightarrow \infty$. Thus, we have:

Theorem 5. As $n \rightarrow \infty$, protocol $p(k, l)$ where $k=(\log n+$ $\left.\frac{1}{2} \log \log n\right)$, and $l=k / 2$ cannot provide authentication for the star network with $n$ nodes. And, as $n \rightarrow \infty$, protocol $p(k, l)$ where $k=\left(\log n+\frac{1}{2} \log \log n+1\right)$, and $l=k / 2$ can provide authentication for the star network with $n$ nodes.

The above results highlight an important aspect of the $p(k, l)$ protocols, i.e., it takes constant time, $O(k)$, for the center node to select the subset of keys, that are given to the satellite node, from the initial pool keys. In other words, the time taken to compute the subset of keys does not depend on the number of nodes in the system but only on the size $k$ of the initial pool of keys stored by the center node.

Theorem 6. The $p(k, l)$ distribution satisfies the constraints of Problem statement 2.1 and 2.3 and hence, solves problem 2.4.

Proof. First, the keys given to the nodes are independent of each other by design. Second, the authentication codes are generated by the center node by directly using the keys and not on a derivative of the keys. From these two points, it is straightforward to note that $p(k, l)$ satisfies the constraints of Problem statement 2.4.

When compared to the key distribution for star network in [11] our key distribution requires lesser number of keys. In [11], the center maintains $2 \log n$ keys and in our key distribution protocol the center only needs to maintain $\log n+O(\log \log n)$ keys. The number of keys stored by the satellite nodes is reduced as well. In [11], each satellite node maintain $\log n$ keys and in our key distribution protocol, each satellite node maintains at most $(\log n) / 2+O(\log \log n)$ keys. This value represents the upper bound for the number of keys maintained by the satellite node. Moreover, the result from the above theorem can be verified for most small values of $n$. In particular, we have verified that $\lceil\log n+\log \log n\rceil$ keys suffice for $5<n<1000$ nodes.

Also, since the above theorem applies for the case where $n \rightarrow \infty$, the natural question is about what happens for small values of $n$. Here, we note that we checked whether $\left\lceil\log n+\frac{1}{2} \log \log n\right\rceil$ keys suffice for $n<50000$. For $2 \leq n \leq 1000$, we found this number to be insufficient for 510 values and for $1000 \leq n \leq 50000$, we found that this number to be insufficient for 20313 values. By contrast, if we consider $\left\lceil\log n+\frac{1}{2} \log \log n+1\right\rceil$ keys then this number suffices for the case where the number of nodes is less than 50000. For illustration, we refer the reader to Figure 2(a).

We would like to note that while the reduction in the number of keys is from $2 \log n$ to $\log n+O(\log \log n)$, this reduction is especially valuable when we consider the number of nodes that can be supported with a given set of keys at the center. For example, Figure 2(b) compares the number of nodes that can be supported with our scheme with that in [11]. If 10 keys are available at the center then the scheme in [11] can support up to 32 satellite nodes whereas our protocol can tolerate upto 252 satellite nodes. Or, if 20 keys are available at the center then the scheme in [11] can support up to 1024 satellite nodes whereas our protocol can tolerate upto 184756 satellite nodes.

\subsection{Optimality of Key Distribution}

Consider the problem of independent key distribution in star network where the number of keys stored by the satellite nodes is equal. Thus, based on the constraints in Section 2, a solution is of the form where, for some values of $k$ and $l$, the center node maintains $k$ keys and each satellite node maintains a subset of size $l$ from that set. In other words, a solution is of the form $p(k, l)$. Therefore, based on Theorem 5 , as $n \rightarrow \infty, \log n+\frac{1}{2} \log \log n+1$ is the optimal number of keys that need to be maintained by the center node.

\section{KEY DISTRIBUTION FOR FULLY CONNECTED COMMUNICATION NETWORK}

We describe how the protocol $p(k, l)$ can be extended to solve Problem 2.4 in all-to-all communication, i.e., where communication graph is fully connected. Thus, this approach would allow any two nodes to communicate securely with each other and any node can broadcast an authenticated message using the keys it has. Our key distribution protocol is designed in stages; the first stage, Stage 1, extends the protocol $p(k, l)$ by considering a fully connected graph as a set of star graphs (or planar graphs). This stage is intended for the case where the number of nodes in the graph is small. Then, Stage 2 uses the scheme in Stage 1 in an hierarchical manner.

Stage 1. In this stage, first, we design a protocol that establishes keys for a group of $d$ nodes. We first select a unique node, say $X$, and consider the star graph where $X$ is the center node and the remaining $d-1$ nodes are satellite nodes. The center node maintains a set of $k=f(d)$ keys, where $f(d)=\left\lceil\log d+\frac{1}{2} \log \log d+1\right\rceil$. Each satellite node receives a unique subset of size $l=f(d) / 2$ from this set. Note that this is an instance of $p(k, l)$ where $k=f(d)$ and $l=f(d) / 2$.

Now, we repeat the above protocol by considering each node as a center node. Thus, for a fully connected network of $d$ nodes, each node is a center node in one star graph and a satellite node in the $d-1$ star graphs. As a center node, each node stores $f(d)$ keys and as a satellite node it stores $(d-1) \cdot f(d) / 2$ keys, one for each of the $d-1$ center nodes. Thus, we have:

Theorem 7. The number of keys stored by each node in Stage 1 is: $f(d)+\frac{d-1}{2} f(d)=\frac{d+1}{2} f(d)$.

Theorem 8. The key distribution in the above solution solves Problem 2.4.

Stage 2. Now, we consider $d$ communication networks from Stage 1 , which gives us a network of $d^{2}$ nodes, and denote this grouping by $G_{p}$. We treat each basic structure of $d$ nodes as a single (virtual) node $U_{i}$ where $1 \leq i \leq d$, i.e., in any key distribution scheme, any key that is given to the virtual node $U_{i}$ is actually given to all the nodes that are part of $U_{i}$.

With this setting, we consider the star graph where $U_{i}$ is the center node for some $1 \leq i \leq d$ and all nodes in $G_{p}-U_{i}$ are satellite nodes. Thus, there are $d(d-1)\left(\approx d^{2}\right)$ satellite nodes. We instantiate $p(k, l)$ for such a network. Thus, with this approach, each node in $U_{i}$ will get $f\left(d^{2}\right)$ keys whereas each node in $G_{p}-U_{i}$ will get $f\left(d^{2}\right) / 2$ keys. Now, we repeat this process by considering the star 


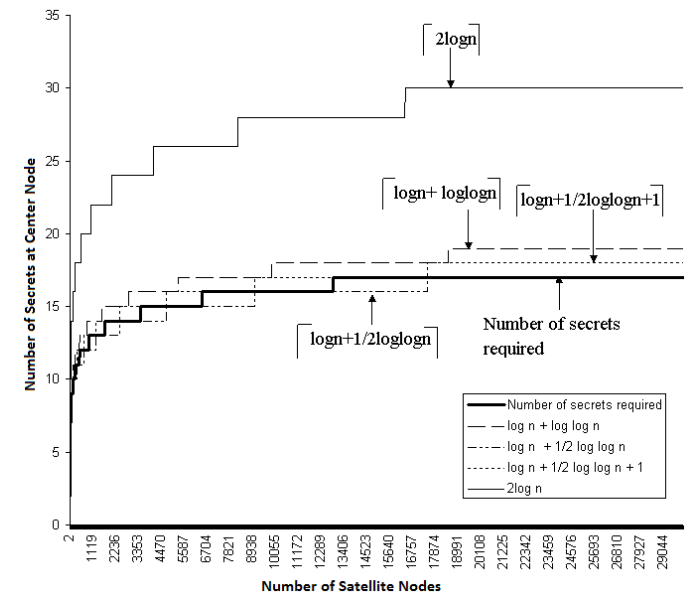

(a) Optimality of Star Network

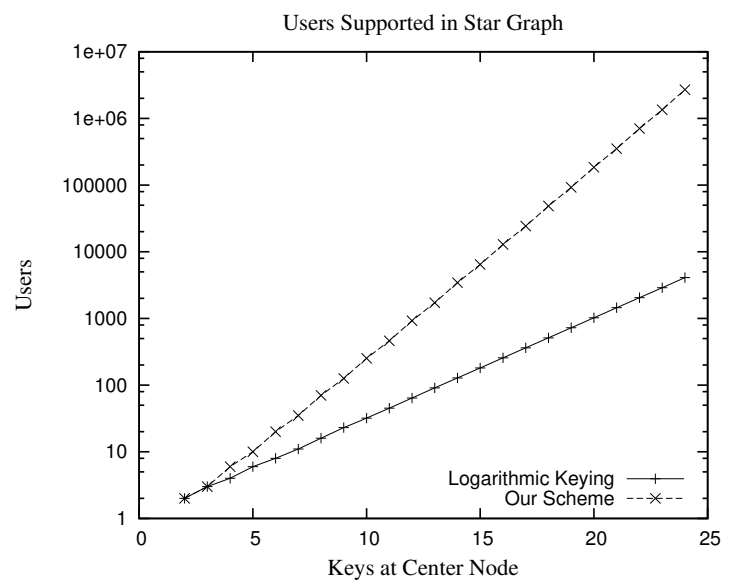

(b) Storage Comparison With [11]

Figure 2: Independent Key Distribution in Star Network

graphs where for each $i, U_{i}$ is the center node. As in Stage 1, each node is a satellite node in $d-1$ star graphs and the virtual node it is in is the center node in one star graph. Thus, each node maintains $\frac{d+1}{2} f\left(d^{2}\right)$ keys in Stage 2 .

For an arbitrary number of nodes $N$, we repeat the protocol of Stage 2 . Thus, at stage $k$ we group $d$ (virtual) nodes considered in stage $k-1$. For $N$ nodes, the number of such stages required will be $\left\lceil\log _{d} N\right\rceil$ to secure communication between all the nodes. The number of keys stored by each node is: $\frac{d+1}{2} \cdot y$ where, the term $y$ is as shown:

$$
y=f(d)+f\left(d^{2}\right)+\ldots+f\left(d^{\left\lceil\log _{d} N\right\rceil}\right)
$$

Theorem 9. The key distribution in the above solution solves Problem 2.4.

\subsection{Storage Analysis}

Now, we evaluate the storage at each node by evaluating formula (1), where $f(d)=\log d+\frac{1}{2} \log \log d+1$.

$$
\begin{aligned}
\mathrm{y}= & \log d+\log d^{2}+\ldots+\log d^{\left\lceil\log _{d} N\right\rceil}+ \\
& O\left(\log \log d+\log \log d^{2}+\ldots+\log \log d^{\left\lceil\log _{d} N\right\rceil}\right) \\
= & \log d+2 \log d+\ldots+\left(\left\lceil\log _{d} N\right\rceil\right) \cdot \log d \\
& +O\left(\log _{d} N \cdot \log \log _{d} N\right) \\
= & \frac{\left(\left\lceil\log _{d} N\right\rceil\right)\left(\left\lceil\log _{d} N\right\rceil+1\right)}{2}(\log d)+O\left(\log _{d} N \cdot \log \log _{d} N\right) \\
= & \frac{\left(\left\lceil\log _{2} N\right\rceil\right)\left(\left\lceil\log _{2} N\right\rceil+1\right)}{2 \log d}+O\left(\log _{d} N \cdot \log \log _{d} N\right)
\end{aligned}
$$

Note that, when evaluating the term $O\left(\log _{d} N \cdot \log \log _{d} N\right)$, we have used some upper bound approximations, such as $O(n !)=$ $O\left(n^{n}\right)$, in the above formula and therefore, the final computed value is higher than the actual value. The storage at each node is given by: $\frac{(d+1)}{2} \cdot y$ where the value of $y$ is substituted from (2). By letting $d=2$, the number of keys per node is: $\frac{3 \log N(\log N+1)}{4}$.

In Figure 3, we compare the per node storage required in our approach with the best case storage cost required in the existing approaches [1, 11, 28]. The approach of Aiyer et al. [1] is denoted Key Grids and has a storage of $4 \log ^{2} n$ keys per node. The approach by Ehab et al. [11] is denoted by Logarithmic Keying and has a storage of $\log ^{2} n$ keys per node. The approach by Novales et al. [28], which is an improvement over [11], is denoted by Parameterized Keying and has a storage of $\frac{3}{2} \cdot\left(\log ^{2} n-\log n\right)+\log n-3$ keys per node. The approach in [28] has higher collusion resistance than the approaches in $[1,11]$. Whereas the approach from [28] uses the approach by Ehab et al. [11] as the base scheme for the star graph and hence, has a higher storage cost than our approach. We note that, by adjusting the parameter $d$ in our approach it is possible to achieve similar bounds on the collusion resistance compared to the approach from [28] while keeping the storage lower than this approach. This is possible due to the optimal storage cost of the star graph as shown in Section 4. From this figure we can see that the storage required using our stage-wise approach is lower than that of previous approaches.

Signature and Verification Costs. Since our protocols use symmetric keys for generating signatures, we have not explicitly 


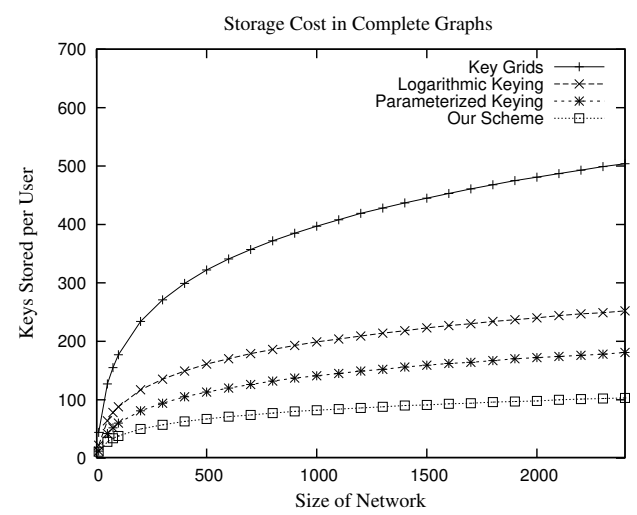

Figure 3: Storage for Fully Connected Networks

shown these costs in this paper as these costs are directly proportional to the storage cost. For the same reason we have not compared these costs against the existing approaches [1, 11, 28].

\subsection{Note on Optimality of Storage}

We note that, the key distribution for star networks, in Section 4, is optimal in terms of storage required. However, it is not known whether the key distribution for fully connected networks, and other networks (described next section) is optimal.

\section{KEY DISTRIBUTION IN ACYCLIC, PLANAR AND COMPLETE BIPARTITE NETWORKS}

In this section, we extend $p(k, l)$ for the cases where the communication graph is acyclic, planar, or complete bipartite. An acyclic network is a network whose topology is an acyclic graph. Similar definitions apply for planar and complete bipartite networks.

\subsection{Acyclic Networks}

In an acyclic network, a node can communicate with only those nodes that have an incident edge to this node. Two nodes not connected by an edge need not communicate with each other. Since an acyclic undirected graph consists of a set of trees, we describe the key distribution for a tree. The same algorithm can be applied for each tree separately to obtain the key distribution for acyclic graphs. Given a tree, we choose one of the nodes in it as a root and consider the corresponding rooted version. In particular, this allows us to define a parent of node in the tree and to define whether a node is a leaf. Now, consider a non-leaf node and its children in this network. This sub-network is a star graph where the non-leaf node is the center node and the children are satellite nodes. Now, we apply the $p(k, l)$ key distribution with appropriate values of $k$ and $l$ for this star graph. We repeat this process for each star graph obtained by considering a non-leaf node and its children. The keys assigned to a node in the tree are the same as the union of the keys assigned to it in any star graph considered in this fashion.

In the star graph, the non-leaf node which is the center node gets $\log d+O(\log \log d)$ keys and each satellite node gets $\frac{1}{2} \log d+$ $O(\log \log d)$ keys, where $d$ is the degree of the center node. Furthermore, for any given non-leaf node, it is a center node in at most one star graph considered above. Likewise, it is a satellite node in at most one star graph (where its parent is the center node) considered above. Thus, the number of keys at any node is at most $\frac{3}{2} \log d+O(\log \log d)$, which is less than that in [11] where $2 \log d$ keys are maintained.

\subsection{Planar Networks}

The extension to planar network is similar to that in [11]. In particular, in [11], a well-known result from graph theory [9], which states that any planar graph $G$ can be decomposed into at most three acyclic graphs, is used. These acyclic graphs are also called factors where each factor has the same nodes as the original graph $G$ and the degree of each factor is at most the degree of the original graph. We use the decomposition technique to distribute keys to the nodes. First, we decompose the graph into $t$ factors, where $1 \leq t \leq 3$. Next, since each factor is an acyclic graph, we instantiate the key distribution for acyclic networks described earlier. One requirement of the key distribution is that, we need to secure each communication channel, i.e., every edge that exists in the original graph. From the properties of factors, we observe that each edge occurs in at most one factor. Thus, each node in the graph receives $t$ times the keys required in a $p(k, l)$ distribution. Note that, the number of keys held by each node can be reduced by reusing them in the different factors. Hence, for key distribution in planar networks, each node stores at most $O(t(\log d+\log \log d))$ keys where $d$ is the maximum degree of the graph $G$.

\subsection{Complete Bipartite Graphs}

A complete bipartite graph is a graph $G(V, E)$ such that its vertex set can be partitioned into two disjoint sets, $V_{1}$ and $V_{2}$, the edge set, $E$ is induced by these two vertex sets such that every vertex in $V_{1}$ is connected to every other vertex in $V_{2}$. No edge exists between any of the vertexes in $V_{1}$ (respectively, $V_{2}$ ). Example of such communication graph is one where the vertex set $V_{1}$ contains servers and the vertex set $V_{2}$ contains clients. The communication is from servers to clients and vice-versa. No server (respectively, client) needs to communicate with each other.

For this network, we employ the following key distribution technique. We treat the vertex set $V_{1}$ as a single center node, say $C$, and the vertex set $V_{2}$ as satellite nodes. As this represents a star network with $C$ as star node, we instantiate a $p(k, l)$ key distribution for this network. Thus, in this distribution, the node $C$ needs to store $\log \left|V_{2}\right|+O\left(\log \log \left|V_{2}\right|\right)$ keys. Since $C$ represents the vertex set $V_{1}$, each of the nodes in $V_{1}$ is given all these keys. We repeat this procedure by treating the vertex set $V_{2}$ as the center node and the vertex set $V_{1}$ as the satellite nodes. This key distribution would provide an additional $\frac{1}{2} \log \left|V_{1}\right|+O\left(\log \log \left|V_{1}\right|\right)$ keys to each node in $V_{1}$. Thus, the number of keys given to nodes in $V_{1}$ is $\frac{3}{2} \log d+O(\log \log d)$, where $d=\max \left(\left|V_{1}\right|,\left|V_{2}\right|\right)$ is the maximum degree of any node in the communication graph.

\section{EVALUATION OF COLLUSION RESISTANCE}

In this section, we evaluate the collusion resistance of our protocols. First, for star networks, we evaluate the collusion resistance gained by choosing a higher number of keys by the center node. Second, for fully connected networks, we show that the overall storage per node increases marginally as the desired level of collusion resistance increases. We considered these two topologies as they represented the two ends of the spectrum of the communication 
topologies. The results for other topologies are similar and we omitted due to space constraints.

We begin with a brief formal analysis for collusion resistance in star network by computing the probability of successful collusion of two nodes in $p(k, l)$ where $l=\frac{k}{2}$ and $k=\log n+\frac{1}{2} \log \log n+1$. Let $U_{t}$ be a target node and $U_{i}, U_{j}$ be the colluding nodes that are attempting to send forged messages to $U_{t}$, posing as the center node. Let $X_{s r}$ denote the random variable for the probability that a key $K_{s} \in K\left(U_{r}\right)$. The collusion is successful if: $K\left(U_{i}\right) \cup K\left(U_{j}\right) \supseteq K\left(U_{t}\right)$. From the results in [10], the expected value of this event is given by:

$$
E\left[\Pi_{s=1}^{s=l}\left(1-\left(1-X_{s i}\right)\left(1-X_{s j}\right) X_{s t}\right)\right]
$$

For $p(k, l)$, with $l=\frac{k}{2}$, we compute the probability $f=P\left(X_{s r}=1\right)$, which is the probability of a key being part of any subset $l$ of $k$. Note that the number of subsets of size $l$ from $k$ is given by $\left(\begin{array}{l}k \\ l\end{array}\right)$, which can be written as: $\left(\begin{array}{c}k-1 \\ l\end{array}\right)+\left(\begin{array}{c}k-1 \\ l-1\end{array}\right)$ where the first term is all possible $l$-sized sub-sets that do not include a given key and the second term is all sub-sets that include the key. When $l=\frac{k}{2}$ these two terms can be shown to be equal and hence, $P\left(X_{s r}=1\right)=f=$ $\frac{1}{2}$. Therefore, the probability of successful collusion is given by: $P($ collusion $) \leq(1-(1-f)(1-f) f)^{l}$. Substituting for $f$, we get $P($ collusion $) \leq(7 / 8)^{l}$. Since $l$ depends on $k$, which in turn depends on $n$, a larger value of $k$ will achieve a higher collusion resistance for the case where $l=\frac{k}{2}$. However, if $l<\frac{k}{2}$, the collusion resistance is higher and we demonstrate this through an informal analysis in the following due to space constraints.

In Figure 4(a), for a star network, we illustrate the increase in storage at the center node for the set of protocols $p(k, k / x)$ where $x=2,3, \ldots, 10$. In this figure, the $x$-axis shows the minimum number of satellite nodes required to compromise the center node keys and the $y$ axis show the corresponding number of center keys required for this level of collusion resistance. In Figure 4(b), we show the effect of collusion resistance on the storage for fully connected networks. Note that the increase in the number of keys for providing increased collusion resistance is small. For example, similar to the approach in [5], which has a collusion resistance of $w+1$ nodes, it is possible to increase the collusion resistance in our scheme without increasing the storage at the nodes. The approach is as follows: the group controller chooses a large degree $d$ for the hierarchy at the lowest level and instantiates $p(k, k / w+1)$. Hence, at the lowest level the collusion resistance is $w+1$ for any given sender. For higher levels of the hierarchy, the group controller chooses $d=2$ and obtains the storage benefits. This construction shows that higher collusion resistance is possible in our approach without increasing the storage considerably.

Comparative Analysis of Collusion Resistance. Consider the scenario where in the number of keys maintained in $p(k, l)$ is equal to those maintained in the approach from [11] (respectively, approaches from $[27,28,45])$. By maintaining a larger set of keys at the center node, it is possible to maintain a smaller set of keys at the satellite nodes while still ensuring that each satellite node receives a unique set. Furthermore, the approach in [11] uses an identifier (ID) based key assignment wherein two nodes with specific IDs can break the scheme. Our approach does not have any structure and thereby, the colluding nodes need to search for suitable nodes to compromise by comparing the key sets they possess. This increases the difficulty of collusion in our scheme when compared to the scheme in [11]. Further, the schemes in [27, 28] are built by using the scheme in [11] as the basis. Hence, the collusion resistance of the schemes in $[27,28]$ is proportional to the collusion resistance of the scheme in [11]. Finally, the scheme in Section 4 has, asymptotically, the same collusion resistance as the scheme in [11] (also, $[27,28]$, which are based on [11]). However, the scheme in Section 4 has better collusion resistance (see Figure 4(a)) when only numbers are used to measure the collusion resistance.

\section{INTERNAL AND EXTERNAL ATTACKS}

In this section, we show that our key distribution protocols can provide a tradeoff between handling internal vs external attacks. The storage at the node can be increased or reduced depending on the likelihood of the possible attacks. An internal attack is launched by two or more colluding satellite nodes that are currently part of the network. These satellite nodes combine their keys and try to compromise all the keys of the center node. The goal of an internal attack is to impersonate the center node and inject false information into the network or tamper the messages sent by the center node. Recall that, in the protocol in [11], two specifically chosen nodes are sufficient to compromise all the center node keys. An external attack is launched by an intruder who is not part of the network but is able to listen on to the communication in the network. The goal of the external attacker is to impersonate one of the nodes in the network, i.e., it wants to send a message and the corresponding authentication codes, to the center node and have it accepted as a genuine message. Also, we are not concerned with replay of genuine packets, as it can be handled using standard techniques.

To illustrate the tradeoff between internal and external attacks, consider two protocols $p(k, l)$, where $l=k / 3$ or $l=2 k / 3$. Since $C(k, k / 3)=C(k, 2 k / 3)$, the number of nodes that can be handled in either protocols is same. Now, consider the effect of these protocol instances on the two types of attacks.

(a) Internal Attacks For $p(k, k / 3)$, the number of keys held by a satellite node is small. In this case, at least three internal attackers must collude to collect all the center keys. By contrast, for $p(k, 2 k / 3)$, the number of keys held by a satellite node is large. And, only two internal attackers can collude to collect all the center keys. Hence, for $p(k, k / 3)$, an internal attacker has to perform more work to complete the attack successfully. This implies that reducing the satellite node storage provides better collusion resistance against internal attacks.

(b) External Attacks For $p(k, k / 3)$, an external attacker only needs to generate $k / 3$ valid authentication codes, but for $p(k, 2 k / 3)$, an external attacker needs to generate $2 k / 3$ valid authentication codes. This issue needs to be considered especially if weak authentication codes are used due to resource constraints, e.g., when only a small number of bytes generated by a hash function are included. For $p(k, 2 k / 3)$, the number of keys held by a satellite node is large. For an external attacker, this key distribution increases the difficulty in compromising all the keys of a particular satellite node. Hence, when $p(k, 2 k / 3)$ is deployed, the external attacker has to perform more work to compromise the satellite node. Thus, increasing the storage at the satellite node provides better resistance against external attacks.

The above discussion shows that our key distribution protocol provides tradeoffs for handling internal and external attacks. These tradeoffs can be generalized as $p(k, k / c)$ and $p(k, k(1-1 / c))$ where $c>1$ where the value of $c$ can be appropriately chosen to achieve the desired attack resilience. 

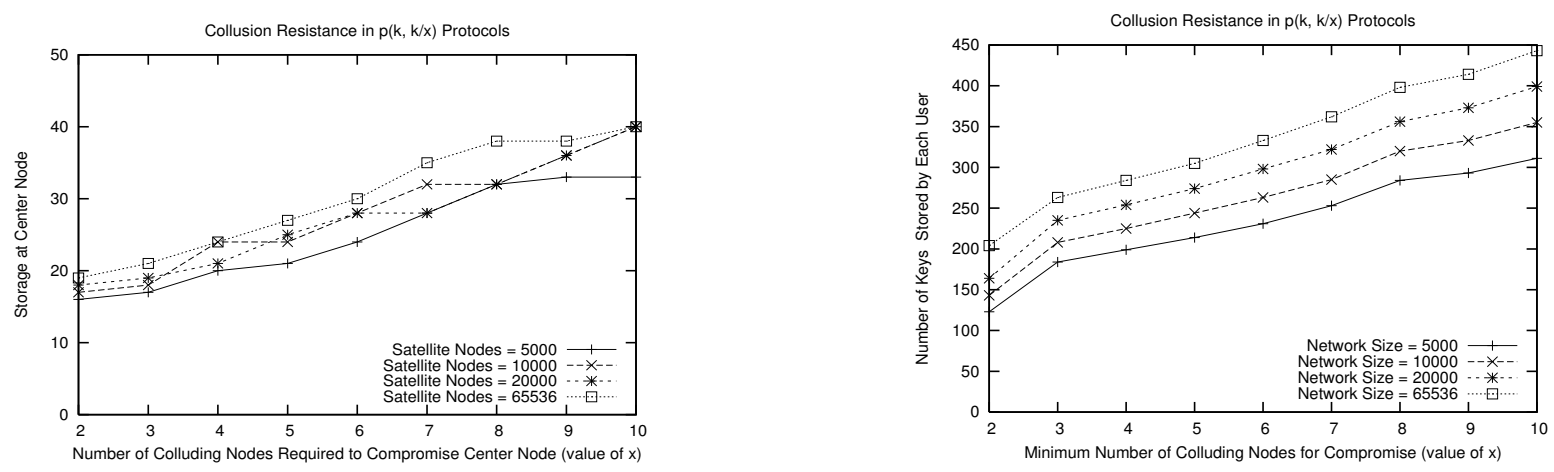

Figure 4: Collusion Resistance in Star and Fully Connected Networks

(a) Star Network

(b) Fully Connected Network

\section{APPLICATION IN SENSOR NETWORKS}

One scenario in sensor networks that requires authentication is reprogramming of sensor nodes. In reprogramming, the base station transmits a new program that needs to be executed in place of the currently running program. Since the base station may not be able to reach all the sensor nodes, the sensors that receive the program instructions propagate them further up in the network. The intermediate sensors are only required to propagate the received instructions without adding or removing any information. Hence, in this scenario, the network is a star network where there is a single sender, the base station, and several satellite nodes in the form of sensors. If the sensor network has multiple base stations then the resulting graph would be bipartite then, each base station communicates with a different part of the network and transmits the program. In this scenario, the network resembles a bi-partite graph where the base stations are in one partition and the sensors are there in the other partition. Therefore, it is important for the sensor nodes to be able to verify that the instructions they receive are indeed sent by (some) base station and that the integrity of the instructions is intact. Hence, protocol such as $p(k, l)$ (or its extension to bipartite graphs) can be used to provide authentication in such sensor networks.

Sensor nodes have limited battery resources and need to reduce the number of transmissions as message transmission is a battery intensive operation. It has been observed that cost of transmitting a single packet using public keys pair is more expensive than transmitting 3-4 KB of data [39]. The additional cost comes from the various public-key operations involved in preparing the data. Using $p(k, l)$, for a group of 1024 sensors, it requires transmission of approximately $5 \mathrm{~KB}$ of data with a 512-bit secure hash function being used for signature generation. The size of the signatures can be further reduced if only a few bits from each signature are included. For instance, if only 384-bits of each signature are included then size of the data reduces by $25 \%$ to $3.75 \mathrm{~KB}$, where the number of bits to include depends on the desired level of security. Since the success of an adversary depends on forging all the sender signatures, the forgery resistance of this curtailed signature scheme can be verified to be secure in most practical situations. A hash functions like SHA256 , which yields 256-bit output, is very secure in most scenarios and hence, can further reduce the signature size in $p(k, l)$. Even if the sensor network utilizes IEEE 802.15.4 for communication, which has an MTU of 127 bytes and payload of 104 bytes, our solutions still provide the smallest possible signature and verification cost when compared with other existing solutions.

\section{CONCLUSION}

In this paper, we considered the problem of independent key distribution for broadcast authentication. Our key distribution is such that it allows each node to provide authentication for unicast communication as well as broadcast communication where it sends a message to all nodes it intends to communicate with. We presented solutions for the cases where the communication graph is a: (1) star graph, (2) acyclic graph, (3) complete bipartite graph and (4) fully connected graph. For the star graph, we presented an optimal lower bound on key distribution with broadcast capability. We showed that this lower bound is indeed achievable and tight. In particular, we showed that as $n \rightarrow \infty, \log n+\frac{1}{2} \log \log n+1$ keys at the center node suffice for virtually all cases of practical interest. Using $p(k, l)$, we showed that it is possible to reduce the keys maintained in an acyclic, planar, complete bipartite and fully connected communication graphs. However, the optimality of the number of keys for these graphs is still an open question. We demonstrated the security of our protocols by analyzing their collusion resistance and considering different attack scenarios. We demonstrated the applicability of our protocols in wireless sensor networks.

One of the future work in this area is to identify lower bounds for keys in different network topologies, which form the core of various communication networks. Another future direction is to explore possibility of applications in Internet-of-Things networks. Such an analysis has been done by Zhao et al. in [48] for the random key distribution protocols [12], but is an open problem for deterministic key pre-distribution protocols.

\section{ACKNOWLEDGEMENT}

The work of Bezawada was supported in part by funds from NSF under Award No. CNS 1650573, CableLabs, AFRL, Furuno Electric Company, SecureNok and National Natural Science Foundation of China Grant 61672156. The work of Indrajit Ray and Indrakshi 
Ray was supported in part by funds from NSF under Award No. CNS 1650573, CableLabs, AFRL, Furuno Electric Company, and SecureNok. The work of Rui Li was supported in part by grant from National Natural Science Foundation of China Grant 61672156.

\section{REFERENCES}

[1] Amitanand Aiyer, Lorenzo Alvisi, and Mohamed Gouda. 2006. Key grids: A Protocol Family for Assigning Symmetric Keys. In Proc. IEEE Int. Conf. on Network Protocols (ICNP). 178-186.

[2] E. Ayday and F. Fekri. 2012. A Secure Broadcasting Scheme to Provide Availability, Reliability and Authentication for Wireless Sensor Networks. Ad Hoc Networks 10, 7 (2012), 1278-1290.

[3] Dan Boneh, Glenn Durfee, and Matt Franklin. 2001. Lower Bounds for Multicast Message Authentication. In Advances in Cryptology (EUROCRYPT). Vol. 2045. 437-452.

[4] Seyit A. Camtepe and Bülent Yener. 2007. Combinatorial Design of Key Distribution Mechanisms for Wireless Sensor Networks. IEEE/ACM Trans. on Networking 15, 2 (2007), 346-358.

[5] Ran Canetti, Juan Garay, Gene Itkis, Daniele Micciancio, Moni Naor, and Benny Pinkas. 1999. Multicast security: a taxonomy and some efficient constructions. In Proc. of the IEEE Joint Conf. on Computer Communications (INFOCOM). 708-716.

[6] Taehwan Choi, Hrishikesh B. Acharya, and Mohamed G. Gouda. 2013. The Bes Keying Protocol for Sensor Networks. Pervasive and Mobile Computing 9, 4 (2013), 564-571.

[7] Jacek Cichon, Zbigniew Golebiewski, and Miroslaw Kutylowski. 2012. From Key Predistribution to Key Redistribution. Theoretical Computer Science 453 (2012) 75-87.

[8] Pedro Cirne, André Zúquete, and Susana Sargento. 2018. TROPHY: Trustworthy VANET routing with group authentication keys. Ad Hoc Networks 71 (2018), 45-67.

[9] C. J Colbourn. 1987. The Combinatorics of Network Reliability. Oxford University Press.

[10] Martin E. Dyer, Trevor I. Fenner, Alan M. Frieze, and Andrew Thomason. 1995 On Key Storage in Secure Networks. Journal of Cryptology 8, 4 (1995), 189-200.

[11] Ehab S. Elmallah, Mohamed G. Gouda, and Sandeep S. Kulkarni. 2008. Logarithmic Keying. ACM Trans. on Autonomous Adaptive Sys. 3, 4 (2008), 1-18.

[12] Laurent Eschenauer and Virgil D. Gligor. 2002. A Key-management Scheme for Distributed Sensor Networks.. In Proc. of the ACM Conf. on Computer and Communications Security (CCS). 41-47.

[13] G. Gaubatz, J.-P. Kaps, E. Ozturk, and B. Sunar. 2005. State of the Art in Ultra-low Power Public Key Cryptography for Wireless Sensor Networks. In Proc. of the IEEE Conf. on Pervasive Computing and Communications (PerCom) Workshops. 146-150.

[14] Li Gong and David J. Wheeler. 1990. A Matrix Key-Distribution Scheme. fournal of Cryptology 2, 1 (1990), 51-59.

[15] Bogdan Groza, Stefan Murvay, Anthony Van Herrewege, and Ingrid Verbauwhede 2017. Libra-can: Lightweight broadcast authentication for controller area networks. ACM Trans. on Embedded Computing Sys. (TECS) 16, 3 (2017), 90.

[16] Kevin J. Henry, Maura B. Paterson, and Douglas R. Stinson. 2013. Practical Ap proaches to Varying Network Size in Combinatorial Key Predistribution Schemes. In Selected Areas in Cryptography (Lecture Notes in Computer Science), Vol. 8282 89-117.

[17] Mahmud Hossain, Ragib Hasan, and Anthony Skjellum. 2017. Securing the Internet of Things: A Meta-Study of Challenges, Approaches, and Open Problems. In Proc. of IEEE Int. Conf. on Distrib. Computing Sys. Workshops (ICDCSW). IEEE, 220-225.

[18] Jean-Pierre Hubaux, Levente Buttyán, and Srdan Capkun. 2001. The Quest for Security in Mobile Ad Hoc Networks. In Proc. of the ACM Int. Symp. on Mobile Ad Hoc Networking and Computing (MobiHoc). 146-155.

[19] Michelle Kendall, Keith M. Martin, Siaw-Lynn Ng, Maura B. Paterson, and Douglas R. Stinson. 2012. Broadcast-enhanced Key Predistribution Schemes. IACR Cryptology ePrint Archive 2012 (2012), 295.

[20] Jun Young Kim, Wen Hu, Dilip Sarkar, and Sanjay Jha. 2017. ESIoT: Enabling Secure Management of the Internet of Things. In Proc. of the ACM Conf. on Security and Privacy in Wireless and Mobile Networks (WiSec). 219-229.

[21] Sandeep S. Kulkarni, Mohamed G. Gouda, and Anish Arora. 2006. Secret instantiation in Ad-Hoc Networks. Computer Communications 29 (2006), 200-215.

[22] Hartono Kurnio, Safavi-Naini Rei, and Huaxiong Wang. 2002. Efficient Revocation Schemes for Secure Multicast. In Proc. of the Int. Conf. on Information Security and Cryptology (ICISC), Vol. 2288. Springer, 160-177.

[23] Jooyoung Lee and Douglas R. Stinson. 2005. Deterministic Key Predistribution Schemes for Distributed Sensor Networks. In Selected Areas in Cryptography. 294-307.

[24] Dongang Liu and Peng Ning. 2003. Efficient Distribution of Key Chain Commitments for Broadcast Authentication in Distributed Sensor Networks. In Proc. of the Network and Distributed System Security Symposium (NDSS).

[25] Yi Mu and Vijay Varadharajan. 1996. On the Design of Security Protocols for Mobile Communications. In Information Security and Privacy. Lecture Notes in
Computer Science, Vol. 1172. 134-145.

[26] Dalit Naor, Moni Naor, and Jeffery Lotspiech. 2002. Revocation and Tracing Schemes for Stateless Receivers. Electronic Colloquium on Computational Complexity (ECCC) (2002)

[27] Ramon Novales and Neeraj Mittal. 2009. TASK: Template-Based Key Assignment for Confidential Communication in Wireless Networks. In Proc. of the IEEE Symp. on Reliable Distrib. Sys. (SRDS). 209-216.

[28] Ramon Novales and Neeraj Mittal. 2011. Parameterized Key Assignment for Confidential Communication in Wireless Networks. Ad Hoc Networks 9, 7 (2011), $1186-1201$.

[29] Maura B. Paterson and Douglas R. Stinson. 2014. A Unified Approach to Combinatorial Key Predistribution Schemes for Sensor Networks. Designs, Codes and Cryptography 71, 3 (2014), 433-457.

[30] Karl Pearson. 1924. Historical Note on the Origin of the Normal Curve of Errors. Biometrika 16 (1924), 402-404.

[31] Adrian Perrig, Ran Canetti, J.D. Tygar, and Dawn Song. 2002. The TESLA Broadcast Authentication Protocol. RSA CryptoBytes 5 (2002).

[32] Adrian Perrig, Robert Szewczyk, J. D. Tygar, Victor Wen, and David E. Culler. 2002. SPINS: Security Protocols for Sensor Networks. Wireless Networks 8, 5 (2002), 521-534.

[33] Di Pietro, L. V. Mancini, Y. W. Law, S. Etalle, and P. J. M. Havinga. 2003. LKHW: A Directed Diffusion-Based Secure Multicast Scheme for Wireless Sensor Networks. In Proc. of the Int. Conf. on Parallel Processing Workshops (ICPP). 397-406.

[34] Rodrigo Roman, Cristina Alcaraz, Javier Lopez, and Nicolas Sklavos. 2011. Key Management Systems for Sensor Networks in the Context of the Internet of Things. Computers \& Electrical Engineering 37, 2 (2011), 147-159.

[35] Rodrigo Roman, Jianying Zhou, and Javier Lopez. 2013. On the Features and Challenges of Security and Privacy in Distributed Internet of Things. Computer Networks 57, 10 (2013), 2266-2279.

[36] Lifeng Sang and Anish Arora. 2008. Spatial Signatures for Lightweight Security in Wireless Sensor Networks. In Proc. of the IEEE Joint Conference on Computer Communications (INFOCOM). 2137-2145.

[37] Ivor Schneider. 2005. Abraham De Moivre, The Doctrine of Chances $(1718,1738$, 1756). Grattan-Guinness, I., Landmark Writings in Western Mathematics 1640-1940 (2005).

[38] Sabrina Sicari, Alessandra Rizzardi, Luigi Alfredo Grieco, and Alberto CoenPorisini. 2015. Security, Privacy and Trust in Internet of Things: The Road Ahead. Computer networks 76 (2015), 146-164.

[39] Arvinderpal S. Wander, Nils Gura, Hans Eberle, Vipul Gupta, and Sheueling Chang Shantz. 2006. Energy Analysis of Public-Key Cryptography for Wireless Sensor Networks. In Proc. of Int. Conf. on Pervasive Computing and Communications (PerCOM). 324-328.

[40] Pan Wang, Peng Ning, and Douglas S. Reeves. 2004. Storage-Efficient Stateless Group Key Revocation. In Proc. of Int. Conf. on Information Security (ISC) (Lecture Notes in Computer Science), Vol. 3225. Springer.

[41] Chung Kei Wong, Mohamed Gouda, and Simon S. Lam. 2000. Secure Group Communications Using Key Graphs. IEEE/ACM Trans. on Networking 8, 1 (February 2000), 16-30.

[42] Chung Kei Wong and Simon S. Lam. 1999. Digital Signatures for Flows and Multicasts. IEEE/ACM Trans. on Networking 7, 4 (1999), 502-513.

[43] Taojun Wu, Yi Cui, Brano Kusy, Akos Ledeczi, Janos Sallai, Nathan Skirvin, Jan Werner, and Yuan Xue. 2007. A Fast and Efficient Source Authentication Solution for Broadcasting in Wireless Sensor Networks. In New Technologies, Mobility and Security. Springer, Dordrecht, 53-63.

[44] Yang Xiao, Venkata Krishna Rayi, Bo Sun, Xiaojiang Du, Fei Hu, and Michael Galloway. 2007. A Survey of Key Management Schemes in Wireless Sensor Networks. Computer Communications 30, 11-12 (2007), 2314-2341.

[45] Y.E. Yang and J.D. Touch. 2008. Protocol Family for Optimal and Deterministic Symmetric Key Assignment. In Proc. of the Int. Conf. on Networking (ICN). 207212.

[46] Xuan Zha, Wei Ni, Kangfeng Zheng, Ren Ping Liu, and Xinxin Niu. 2017. Collaborative Authentication in Decentralized Dense Mobile Networks with Key Predistribution. IEEE Trans. on Information Forensics and Security 12, 10 (2017), 2261-2275.

[47] Junqi Zhang and Vijay Varadharajan. 2010. Wireless sensor network keymanagement survey and taxonomy. Fournal of Network and Computer Applications 33 (2010), 63-75.

[48] Jun Zhao. 2017. Probabilistic key predistribution in mobile networks resilient to node-capture attacks. IEEE Trans. on Information Theory 63, 10 (2017), 6714-6734.

[49] Yun Zhou and Yuguang Fang. 2006. BABRA: Batch-based Broadcast Authentication in Wireless Sensor Networks. In Proc. of the IEEE Global Telecommunications Conf. (GLOBECOM).

[50] Sencun Zhu, Sanjeev Setia, Shouhuai Xu, and Sushil Jajodia. 2006. GKMPAN: An Efficient Group Rekeying Scheme for Secure Multicast in Ad-Hoc Networks. Journal of Computer Security 14, 4 (2006), 301-325.

[51] Jan Henrik Ziegeldorf, Oscar Garcia Morchon, and Klaus Wehrle. 2014. Privacy in the Internet of Things: Threats and Challenges. Security and Communication Networks 7, 12 (2014), 2728-2742. 